Archives de sciences sociales des religions

$177 \mid 2017$

Mondes juifs en mouvement

\title{
Sanctifier le cloître
}

Les dons de reliques aux carmélites de Lyon et Rouen à l'époque moderne

Sanctification of the cloister. Gifts of relics to the Carmelites of Lyon and Rouen in the modern period

Santificar el claustro. reliquias de donaciones a Carmelite Lyon y Rouen en la era moderna

\section{Nicolas Guyard}

\section{(2) OpenEdition}

\section{Journals}

Édition électronique

URL : http://journals.openedition.org/assr/29363

DOI : $10.4000 /$ assr.29363

ISSN : $1777-5825$

Éditeur

Éditions de l'EHESS

Édition imprimée

Date de publication : 1 mars 2017

Pagination : 293-312

ISSN : 0335-5985

Référence électronique

Nicolas Guyard, "Sanctifier le cloître », Archives de sciences sociales des religions [En ligne], 177 | 2017, mis en ligne le 01 mars 2019, consulté le 06 janvier 2021. URL : http://journals.openedition.org/assr/ 29363 ; DOI : https://doi.org/10.4000/assr.29363 


\section{Nicolas Guyard}

\section{Sanctifier le cloître}

\section{Les dons de reliques aux carmélites de Lyon et Rouen à l'époque moderne}

En 1626, Anne de Souvré, abbesse de Saint-Amand et de Préaux, écrit une courte lettre à la prieure du carmel de Rouen, accompagnant des reliques de saint Gérin :

Madame, tres humble Salut en nre seigr,

Je vous de mande pardon davoir sy lhontans manque amaquiter de la promesce que ie vous avois faite de vous en voier de nos sainte reliques qui sont tres asurer les exquirians sont sur chaque ossemens.

Nous vous suplirons madame que nous puissions avoirpart à vos sainctes prieres et que me croyes sans fin.

Votre trs humble seur trs affeccionnée voysine et servante en nre seigneur ${ }^{1}$.

Cet exemple met en scène deux membres importants de la vie religieuse rouennaise, au début du XVII ${ }^{\mathrm{e}}$ siècle. Anne de Souvré, abbesse élue en 1620, réforme l'abbaye de Saint-Amand de Rouen, durement touchée par les troubles religieux de la fin du $\mathrm{XVI}^{\mathrm{e}}$ siècle, tout en accumulant un important trésor de reliques $^{2}$. Les carmélites, installées à Rouen depuis 1609, incarnent alors le renouveau monastique féminin dans la ville, soutenues par le clergé local et les élites parlementaires de la ville. Différents liens ici se nouent. Le premier est matériel: un couvent demande à un autre un fragment d'une relique désormais partagée. Le second est spirituel: des dévotions communes peuvent se fixer autour du corps saint et les carmélites prient, comme l'a demandé l'abbesse, pour leurs consœurs de Saint-Amand. Le troisième est social. Le don de relique est une relation entre deux milieux fermés car cloîtrés. La correspondance sanctionne une bonne relation de voisinage, puisque les deux monastères ne sont distants que de quelques dizaines de mètres.

1. Archives Départementales de la Seine-Maritime (désormais A.D.S.M.), 64H13, Reliques des carmélites de Rouen. La lettre est signée "sr Anne Souvré, abaisse indigne ».

2. L'abbaye amasse un trésor de près de 120 reliques tout au long du XVII ${ }^{\mathrm{e}}$ siècle. Voir A.D.S.M., $55 \mathrm{H} 20$. 
Autant de dynamiques qui sont au cœur des arrivées progressives et massives de reliques dans les carmels de Lyon et Rouen. Car cet exemple liminaire n'est pas un cas isolé dans l'ordre fondé par Thérèse d'Avila, acteur majeur de la circulation des corps saints dans la catholicité moderne ${ }^{3}$. Ces deux exemples français ${ }^{4}$ témoignent de l'importance de ces échanges dans la catholicité du XVII ${ }^{\mathrm{e}}$ siècle, dans une véritable économie du sacré, mobilisant des réseaux divers, avec des finalités autant sociales que spirituelles ${ }^{5}$. Ces processus d'accumulation et de concentration du sacré ont surtout été étudiés pour la période médiévale 6 . Peu de travaux ont été consacrés à ce sujet pour l'époque moderne ${ }^{7}$, même si ces circulations commencent à être de mieux en mieux connues, grâce notamment aux deux grandes enquêtes collectives du Centre d'Anthropologie Religieuse Européenne (CARE, aujourd'hui CéSor) sur les corps saints ${ }^{8}$.

Le Grand Siècle apparait comme un temps fort dans la circulation de reliques $^{9}$, dont certaines ont terminé leur parcours dans les carmels de Rouen et Lyon. Ce processus n'est bien sûr pas propre à l'ordre du Mont-Carmel. D'autres ordres religieux, principalement ceux fondés dans la mouvance du Concile de Trente, ainsi que les églises séculières, ont été concernés ${ }^{10}$. Mais les carmélites se distinguent par la quantité et la diversité du trésor ainsi que par la pluralité des réseaux d'approvisionnement en corps saints. Pour obtenir des reliques, les carmélites ont bénéficié de nombreux dons, mode d'échange intrinsèquement lié à la nature et au statut des restes saints, qui, en droit, ne peuvent être vendus. Ces dons ont pu être spontanés ou bien provoqués par

3. Les historiens n'ont guère insisté sur les aspects matériels des dévotions de l'ordre (Hours, 1987; Morgain, 1995; Hours, 2001; Roullet, 2015). D’autres carmels ont accumulé un trésor de reliques important, comme celui de Pontoise, Metz, Toulouse ou celui du faubourg Saint-Jacques à Paris (Eriau, 1929: 106-111).

4. Ces deux couvents carmélites sont particulièrement documentés. Le dossier 64H13 aux A.D.S.M. contient une importante documentation archivistique pour le cas rouennais, alors que l'ouvrage de Jacques-Jules Grisard (Grisard, 1887) contient une documentation sur les reliques absente des archives publiques.

5. Sur ce sujet, une étude pionnière a été menée pour la période médiévale par Patrick J. Geary (Geary, 1986). Katie A. Harris, plus récemment, a mené un travail similaire pour l'époque moderne (Harris, 2014).

6. Edina Bozoky (Bozoky, 2007) et John Osborne (Osborne, 1999) ont étudié ce phénomène en tant que politiques royales et diplomatiques. Patrick H. Geary (Geary, 1990) et Pierre-Vincent Claverie (Claverie, 2009) ont étudié les modalités des circulations, à travers les pratiques du vol, de l'achat et du don. Enfin, Philippe Cordez a étudié la constitution des trésors d'église (Cordez, 2016).

7. Un des cas le mieux documenté reste la collection de Philippe II à l'Escurial (Lazure, 2009 ; Diez, 2005). Sur le don, voir l'article de Maddalena Gana (Gana, 2005).

8. Un premier ouvrage collectif a ouvert de nombreuses pistes de recherche (Boutry, Fabre et Julia, 2009), reprises par l'enquête sur les corps saints des catacombes de Rome (Baciocchi, Duhamelle, 2016).

9. Françoise Le Hénand a donné un aperçu des translations en France au XviI ${ }^{\mathrm{e}}$ siècle (Le Hénand, 2009).

10. Deux exemples dans les villes étudiées: les récollets du couvent de Lyon obtiennent 127 reliques entre 1628 et 1673 ; l'église paroissiale Saint-Vivien de Rouen amasse un trésor de 34 reliques entre 1670 et 1708 . 
une demande des deux couvents, comme le laisse entendre la lettre d'Anne de Souvré. De nombreux individus et communautés ont donné aux carmels de Lyon et Rouen un des objets les plus précieux et recherchés du monde catholique, par l'entremise d'un don qui ne laisse pas entrevoir de contrepartie, tout au moins immédiate. Une question demeure, simple dans sa formulation: pourquoi donner des reliques à ces deux couvents ? L'étude des motivations des donateurs et des carmélites permet de comprendre les enjeux sociaux et dévotionnels autour de cette pratique massive, à travers la constitution du trésor sacré, et la valeur du geste du don dans les sociétés urbaines du XVII ${ }^{\mathrm{e}}$ siècle.

\section{La constitution du trésor sacré. L’accumulation}

\section{Où trouver des reliques à donner?}

Comme tous les nouveaux ordres religieux qui s'installent en ville, les carmélites, lorsqu'elles fondent leur couvent à Rouen en 1609 et Lyon en 1616 , ont besoin de reliques pour sanctifier leur chapelle conventuelle. Tout au long de la période étudiée (jusqu'en 1720), les deux carmels en reçoivent 250. Différentes catégories de reliques apparaissent, renvoyant à des circulations et des temporalités différentes.

La première catégorie, la plus massive, est constituée par les corps saints des catacombes de Rome. Elle représente plus des deux tiers des reliques arrivant dans ces couvents (168 sur 250). Ces reliques catacombaires ont été diffusées dans toute la catholicité moderne, après la (re)découverte des catacombes de Rome en $1578^{11}$ (Baciocchi, Duhamelle, 2016: 11-12). À partir de la fin du XVI ${ }^{\mathrm{e}}$ siècle, Rome et ses sous-sols deviennent donc le principal «centre de production » de reliques (Geary, 1986: 181). Les archives vaticanes témoignent de 37000 restes saints envoyés dans la catholicité ${ }^{12}$. La réception de ces reliques des catacombes dans ces carmels suit peu ou prou la diffusion générale, avec un point culminant entre 1670 et 1700 . Elles constituent alors plus des trois quarts des reliques données. Les couvents carmélites sont un réceptacle parmi d'autres. À Lyon, ce sont dix-neuf établissements religieux, réguliers, séculiers, confréries, qui reçoivent 240 corps saints des catacombes jusqu'à la fin du XVIII siècle. À Rouen, vingt-quatre sanctuaires ont été durant la même période la destination finale de quelques 281 reliques catacombaires. Les carmélites, en obtenant ce premier type de corps saints, participent donc à un vaste mouvement qui concerne toute la catholicité (Baciocchi, Duhamelle, 2016).

11. Les premiers résultats de l'enquête du CARE sur ces reliques ont été publiés dans un article collectif (Baciocchi, Boutry, Duhamelle, Fabre, Julia, 2010), puis dans un livre restituant les résultats de l'enquête (Baciocchi, Duhamelle, 2016). Pour la période médiévale, voir l'article de Massimiliano Ghilardi (Ghilardi, 2003) et celui, pionnier, de Philippe Boutry pour la période contemporaine (Boutry, 1979). Pour les conséquences de la découverte, voir l'article de Roberto Giordani (Giordani, 2007).

12. Ce chiffre doit être considérablement augmenté si l'on prend les archives perdues ou inconnues à ce jour. 
La deuxième catégorie est constituée des reliques découvertes avant le $\mathrm{XVII}^{\mathrm{e}}$ siècle, comprenant les restes des saints locaux, des saints de l'Évangile et des saints médiévaux. Ces reliques sont généralement données par des églises ou couvents anciens, constituant déjà un trésor reliquaire important. Par exemple, le carmel de Rouen reçoit de nombreuses reliques en provenance d'abbayes normandes relativement anciennes, comme celle de Jumièges, de Fécamp, du Bec ou de Préaux. Ces différentes reliques ont été inventées ou reçues au cours des différents temps forts de circulations de corps saints, principalement aux IX ${ }^{\mathrm{e}}, \mathrm{XI}^{\mathrm{e}}$ et XIII ${ }^{\mathrm{e}}$ siècles (Geary, 1986: 178-179). Le don prend ici la forme d'une diffusion par morcellement, de petites particules de reliques. Dans cette deuxième catégorie, plusieurs réseaux spécifiques de diffusion apparaissent, comme par exemple celui des reliques de sainte Ursule et des onze mille vierges martyres de Cologne (Legner, 2003; Moulinier, 1992). Le carmel de Rouen reçoit en 1617, en provenance de l'Abbaye de Fécamp, un os d'une des onze mille vierges, complété en 1624 par un autre don fait par la prieure du carmel de Louvain ${ }^{13}$. Le couvent de Lyon reçoit lui en 1629 une côte de "Saincte Benoite, du nombre des onze mil vierges " (Grisard, 1887: 168). Cette relique, à l'origine, avait été donnée en 1288 depuis Cologne à Jean de Picquigny, vidame d'Amiens, patron fondateur de la collégiale Saint-Martin de Picquigny. Trois siècles et demi plus tard, Honoré d'Albert, duc de Chaulnes et vidame d'Amiens, fit prélever quelques ossements saints, dont une côte offerte au carmel d'Amiens en 1628. La mère prieure de ce couvent envoie l'année suivante une particule des os de la sainte au monastère lyonnais.

Une troisième et dernière catégorie correspond aux reliques des saints canonisés à l'époque moderne, objets d'une diffusion spécifique. Les reliques des saints du carmel ont bien sûr été mises en avant par l'ordre. Le couvent de Rouen pouvait s'enorgueillir de posséder un morceau du voile de la fondatrice Thérèse d'Avila, offert par Jean de Brétigny en 1615. La première prieure, Isabelle des Anges, espagnole, donne également une particule d'os de la sainte la même année. À Lyon, le carmel obtient en 1706 des reliques de la sainte fondatrice données par le père minime Francoeur, ancien prieur du couvent romain de la Sainte-Trinité, qui les avait reçues à Rome auprès de Nicolas Poplauski, évêque de Livonie (Grisard, 1887: 1673). Une autre relique de Thérèse accompagnée d'un os de saint Jean de la Croix arrive au carmel lyonnais, en 1732 après un parcours sinueux. Le couvent des carmélites déchaussées de Barcelone offre en 1731 une parcelle de ses reliques de la sainte à l'évêque de Gérone, Baltasar Bastero. Celui-ci reçoit une demande de la part du nouveau gouverneur de Bellegarde-en-Roussillon, François de Valadous, qui souhaite offrir cette relique insigne à une carmélite lyonnaise, «Madame Gautier». L'évêque procède donc à une nouvelle fragmentation pour satisfaire la demande. La relique arrive finalement une année après, après être passée entre de nombreuses mains (Grisard, 1887: 174-175). Ce

13. A.D.S.M., $64 \mathrm{H} 13$. 
don n'est pas quelconque. En effet, «Madame Gautier» défraie la chronique par sa conversion subite, alors qu'elle menait une carrière de comédienne depuis 1692. Elle est notamment sociétaire au Théâtre-Français en 1716. En 1722, elle décide de changer de vie et entre au carmel de Lyon sous le nom de Marie-Jeanne Augustine de la Miséricorde. La cérémonie est personnellement dirigée par François-Paul de Villeroy, archevêque de Lyon (Grisard, 1887: 137-139). Enfin, autre saint important pour l'époque moderne, les reliques de François de Sales sont envoyées directement depuis Annecy au carmel de Lyon par Joseph-Nicolas Deschamp de Chaumont, évêque de Genève, en 1752 (Grisard, 1887: 173).

Il existe donc à l'époque moderne des lieux de production et des réseaux d'acheminement de reliques. Comment les carmélites, religieuses cloitrées, les ont-elles intégrées?

\section{Obtenir des reliques : circulations et réseaux}

La constitution d'un trésor reliquaire relève d'une « politique » (Bozoky, 2006: 10), terme jusque-là appliqué dans l'historiographie au pouvoir laïc en quête de corps saints. La construction d'un tel ensemble apparait cependant très empirique. Les carmélites saisissent les occasions qui se présentent à elles pour obtenir des reliques, au gré des relations nouées avec des personnalités, des institutions religieuses internes et externes à l'ordre. Ce sont ces relations sociales qui leur ont permis de s'intégrer au sein des réseaux de circulations des corps saints à l'époque moderne. La chronologie des arrivées correspond à deux temporalités parallèles, celle de l'histoire particulière de ces deux carmels dans leur contexte urbain respectif, et celle des dynamiques propres à la catholicité dans son ensemble. L'articulation de cette double temporalité est l'objet de tactiques de la part des carmélites (Certeau, 1990: 59-63). En effet, ces dernières semblent régulièrement jouer sur des occasions d'obtenir des reliques pour leur propre sanctuaire au fil des relations nouées et surtout au gré des opportunités à liées à la chronologie des centres de production et de diffusion.

Différents types de relations apparaissent lors des arrivées de reliques. L'étude de la trajectoire de ces corps saints par les acteurs qui les font circuler montrent divers registres à l'œuvre, souvent complémentaires. Le premier type de relation sociale dans le don de relique est celui du lien ecclésiastique. De nombreux ordres monastiques donnent des reliques au nom d'une parenté et proximité spirituelle, matérielle et géographique. Ce n'est guère étonnant de retrouver de tels acteurs qui ont un accès facilité ou même parfois contrôlent les grands centres de distribution de reliques, en particulier Rome. Le second type de lien social est celui de patronage. Ce terme désigne ici la relation entre un grand personnage, revendiquant une forme de noblesse, et un couvent carmélite. Le don de relique témoigne alors d'une forme de libéralité (Davis, 2003 : 30) qui honore le donateur, tout en faisant la satisfaction du carmel. Le troisième type est un lien familial. Certaines familles offrent des reliques 
au carmel dans lequel réside leur fille. Ce don est autant fait à une personne qu'à une institution. Il sanctionne à la fois la réussite familiale et l'élection sacrée du couvent. Ces trois types qui apparaissent ici ne sont pas exclusifs. Ils peuvent se conjuguer. Ainsi un ecclésiastique peut donner une relique à un carmel où réside une de ses nièces, le réseau ecclésiastique jouant alors tout autant que le lien familial. Ces types de relations n'apparaissent pas de manière uniforme dans la chronologie de la constitution d'un trésor reliquaire. Ils s'inscrivent dans une histoire de la structuration et de la diversification des réseaux propres à la temporalité de chaque carmel.

Deux temps forts se dégagent dans les arrivées de reliques, entrecoupés par un creux de vague. La première période concerne le temps de la fondation du carmel jusqu'à la normalisation de son fonctionnement, généralement marquée par la consécration de l'église du monastère, et la fin des travaux de construction. Le carmel de Rouen est fondé en 1609, celui de Lyon en 1616. Ce temps de création doit se comprendre dans le contexte urbain du premier tiers du XVII ${ }^{\mathrm{e}}$ siècle en France, période de constitution de la "ville monastère » (Lignereux, 2003 : 719). Depuis la fin des guerres de religion jusqu'aux années 1640, de nombreux ordres sont apparus en ville. À Rouen, ce sont près de dix-sept nouvelles maisons religieuses qui sont fondées (Mollat, 1979: 195). À Lyon, 39 maisons masculines et 36 maisons féminines s'installent en ville (Lignereux, 2003 : 720). S'intégrer dans la ville, dans un contexte de concurrence entre ordres religieux, nécessite alors l'appui de puissants fondateurs, laïcs ou ecclésiastiques, permettant aux carmélites d'obtenir la nécessaire permission municipale. Les conseils de ville sont parfois réticents à accorder le droit d'installation, car ces nombreux ordres, souvent mendiants, risquent de ne pas pouvoir subvenir à leurs besoins grâce à la seule quête. Une autonomie financière basée sur des rentes et autres possessions foncières ${ }^{14}$ est requise. Dans le cas des deux carmels, les figures fondatrices sont caractérisées par leur proximité avec le pouvoir royal et par une assise locale importante. Et dans les deux cas, les fondateurs font don d'une relique illustre. À Rouen se détache la figure de Jean de Quintanadoine, sieur de Brétigny, qui joue un rôle important dans le développement de l'ordre du Mont-Carmel en France et à Rouen. Originaire de Burgos en Espagne par son père, il est né à Rouen dans un milieu marchand déjà bien intégré à la bourgeoisie locale ${ }^{15}$. Au cours d'un voyage à Séville en 1583, il visite le couvent des carmélites. Marqué par cette rencontre avec l'ordre, il œuvre ensuite pour l'introduction de l'ordre en France auprès du roi Henri III (Morgain, 1996: 27-30 et Benedict, 1981 : 197-198). C'est lui qui obtient de carmélites espagnoles ayant connu Thérèse d'Avila une relique du voile de la sainte fondatrice de l'ordre (Serouet, 1971 : 88-89), donnée au carmel de Rouen en 1615. Son don est complété

14. À Lyon, par exemple, des rentes sont constituées dès 1615. Voir Archives Départementales du Rhône (désormais A.D.R.), 38H13-14, rentes et pensions du carmel de Lyon.

15. Son père a notamment essayé de sauver les reliques de la Cathédrale Notre-Dame de Rouen durant la prise de la ville par les Huguenots en 1562. Voir à ce sujet A.D.S.M., G 3666. 
la même année par celui d'Isabelle des Anges, première prieure, qui donne une particule d'os de la même sainte obtenue du couvent de Salamanque ${ }^{16}$. Ce double don renvoie à une fondation spirituelle du couvent, désormais rattachée au temps sacré des origines de l'ordre.

À Lyon, le carmel reçoit un don de relique similaire, effectué par le patron fondateur laïc du couvent, Charles de Neufville de Villeroy, gouverneur de la ville pour le roi (Baciocchi, Bonzon, Julia, 2016: 444-449). Son épouse, Jacqueline de Harlay, contribue à fonder le monastère de Lyon en 1616, deux ans avant son décès (Martin, 1908: 80). Elle avait souhaité introduire l'ordre réformé du carmel à Lyon après avoir rendu visite à sa sœur, carmélite au couvent de Paris ${ }^{17}$ (Vachet, 1895 : 214). Charles de Neufville, par le don d'une relique au carmel, honore ainsi la mémoire de son épouse et accomplit dans le même temps un acte d'évergétisme. Ce personnage maitrise les circuits des reliques des catacombes depuis son séjour à Rome en 1605 en qualité d'ambassadeur exceptionnel du roi Henri IV (Lignereux, 2003: 275). En 1612, il donne au collège des jésuites de Lyon le corps de saint Gabin, ramené de Rome (Lignereux, 2003: 408). Il donne également un os de saint Rosalie au carmel du Faubourg Saint-Jacques à Paris (Eriau, 1929: 109). Et en 1628, il demande au chapitre cathédral de Saint-Jean de Lyon d'accorder aux carmélites de la ville un des six corps saints rapportés par le défunt archevêque Denis Simon de Marquemont, après une ambassade exceptionnelle auprès du Pape en 1623 (Grisard, 1887: 160). Le carmel reçoit, lors d'une cérémonie officielle le 12 février 1628, le corps de saint Faustin ${ }^{18}$. Ces deux exemples mettent en scène une relation de patronage. Les fondateurs, par leur rayonnement qui dépasse les simples murs de la cité, se caractérisent par leur capacité, exceptionnelle à l'époque, à obtenir des reliques et à les donner selon leur volonté propre.

Dans les deux cas lyonnais et rouennais, lors de ce temps de fondation, les carmélites sollicitent également des ordres religieux dont elles sont proches. Les autres couvents carmes font parvenir des reliques dans les nouveaux carmels : ce sont les arrivées des reliques des onze mille vierges de Cologne, en 1624 à Rouen et en 1629 à Lyon, en provenance des fondations de Louvain et d'Amiens, créées respectivement en 1611 et 1606 . Ces dons sont complétés par ceux d'ordres ecclésiastiques proches des carmélites. Par exemple, le couvent feuillant de Saint-Bernard de Paris accompagne la fondation du carmel rouennais, s'endettant pour fonder le nouveau monastère. Le père Jean de Saint-François, prieur, envoie en 1607 un premier don, puis un second

16. A.D.S.M., 64H13.

17. Il s'agit de Charlotte de Harlay de Sancy, qui finance la fondation du carmel de Pontoise, alors que Charles de Neufville est gouverneur de la ville (Mellot, 1994: 41).

18. Outre le corps de saint Faustin, Denis Simon de Marquemont reçoit en 1624 les corps de saint Jovin, donné à la chapelle de l'hôpital de la Charité de Lyon, de saint Théodore, saint Léon, saint Porphyre, saint Pontain et saint Anasthase, donné à Mme de Chevrière qui le transmet au couvent des Annonciades Bleues Célestes de Lyon. 
en 1617 alors qu'il est provincial de l'ordre (Pierre, 2006: Annexe III). Un réseau ecclésiastique plus régional est également mobilisé, celui des abbayes normandes, qui disposent au début du XVII ${ }^{\mathrm{e}}$ siècle d'un trésor reliquaire considérable. Le don s'effectue grâce à l'intermédiaire d'ecclésiastiques rouennais, possédant également une charge dans ces monastères, comme Anne de Souvré ou le père Gatteau, qui donne quatre reliques en provenance de l'abbaye de Fécamp en $1617^{19}$.

Cette première période de dons de reliques est ainsi caractérisée par le désir de combler un manque de sacralité dans des carmels nouvellement fondés. Fondateurs et milieux ecclésiastiques s'activent pour fournir le matériau nécessaire, dans des relations de patronage et de proximité ecclésiastique.

Un deuxième grand moment d'arrivées de reliques débutera vers 1670 . Avant cette date, diverses occasions permettent toutefois aux carmélites d'obtenir des reliques. Les relations de patronage sont ici encore au cœur des donations. De grandes figures prestigieuses de l'époque font parvenir des reliques aux carmels. Le monastère lyonnais reçoit par exemple en 1642 une parcelle de la Vraie Croix, donnée par le père Philippe de l'Oratoire, confesseur de la reine d'Angleterre Henriette-Marie, relique prélevée dans la chapelle royale. Puis le couvent reçoit en 1652 et 1656 deux dons de Lucrezia et Carlo Barberini, petits-neveux d'Urbain VIII, dont la famille obtient et distribue de nombreuses reliques romains (Ticchi, 2016: 203). Ils sont présents à Lyon ${ }^{20}$ en 1652 (Grisard, 1887: 167-168). Lucrezia est proche des milieux carmélites, car sa tante habite le couvent de Modène. Elle a donc pu tout à fait visiter le carmel de Lyon lors de sa présence dans la ville.

Ces grands donateurs sont aussi présents, à la même époque, à Rouen, notamment autour du milieu des missions étrangères. Luc Fermanel, issu d'une famille rouennaise, directeur du séminaire des missions étrangères, donne en 1655 des reliques de saint Hyppolite et saint Candide, obtenues directement auprès du Pape Innocent X (Baudry, 1875 : 96). Puis François Pallu, tout juste promu évêque d'Héliopolis et surtout vicaire apostolique de l'administration de la Chine de l'Ouest, donne à son tour au carmel, en 1658, des reliques de saint Marcelin et saint Flavien, puis un corps entier en 1670. Proche des carmélites chez qui il avait fait une retraite à Tours en 1651 (Baudiment, 2006: 30), ce prélat se distingue par une politique de don de corps saints très organisée. Ayant reçu six corps à Rome du cardinal vicaire Ginetti et de Tommaso Candido ${ }^{21}$, il les distribue à différentes institutions ecclésiastiques, renforçant des liens préexistants: le chapitre Saint-Martin de Tours dont il est chanoine, le couvent de la Visitation à Paris, et le carmel de Rouen (Baudiment, 2006: 66-67). Enfin, la duchesse de Longueville, dont l'époux est gouverneur de Normandie, donne

19. A.D.S.M., 64H13, inventaire de 1626.

20. Carlo Barberini aurait notamment loué la maison des Oratoriens de Lyon (Martin, 1909: 250). Selon l'acte de donation, Lucrezia Barberini séjourne rue de la Monnaie (Grisard, 1887: 168). 21. Tommaso Candido, d'origine vénitienne, est très actif à Rome pour obtenir et distribuer des reliques (Duhamelle, 2016). 
en 1659 une parcelle du corps de saint Hildevert, prélevée dans le trésor de l'Abbaye de Gournay, patronnée par son mari²2.

Si ces grands donateurs ne sont plus les fondateurs des deux carmels, ils entretiennent des liens particuliers avec les carmélites et font preuve d'une libéralité qui s'explique en grande partie par leur accès privilégié aux lieux détenant et diffusant des reliques, comme la cour pontificale.

Les réseaux ecclésiastiques sont toujours présents lors de cette période de creux. Le carmel lyonnais envoie en 1651 quatre reliques prélevées en partie dans le trésor de l'abbaye d'Ainay aux carmélites de Rouen. Celles-ci reçoivent également quinze reliques tirées des catacombes romaines, apporté par Philippe Meunier, prêtre de la Compagnie de Jésus ${ }^{23}$.

Ainsi, jusqu'en 1670, les dons de reliques demeurent-ils des événements exceptionnels dans le quotidien du carmel, qui mettent en scène la libéralité des grands donateurs et la solidarité des réseaux ecclésiastiques, qui diffusent les corps saints en les partageant.

Le second temps fort dans les arrivées de reliques aux carmels de Lyon et Rouen s'étend donc entre 1670 et 1720 . Ce sont alors 179 reliques qui densifient les trésors, soit près des trois quarts du total. Différents facteurs expliquent un tel essor. Le premier renvoie au contexte global de la catholicité. C'est de l'achèvement de la réglementation en matière de corps saints, qui permet une diffusion massive et contrôlée. En 1672, le pape Clément X publie un décret de la constitution Ex Commissae, qui règlemente l'extraction et la distribution des reliques des catacombes. À l'échelle du royaume de France, les reliques prennent une signification plus militante après la révocation de l'édit de Nantes en 1685. Les corps saints assument alors pleinement le statut d'armes contre le protestantisme. Enfin, durant cette période, les couvents carmélites connaissent une activité importante. À Lyon, une nouvelle chapelle est consacrée en 1680 par l'archevêque Camille de Neufville de Villeroy (Grisard, 1887: 102). Le carmel est alors dirigé par Éléonore de Villeroy, descendante $\mathrm{du}$ fondateur lyonnais, attirant ainsi d'autres reliques. Les couvents des deux villes recrutent de nombreuses novices dans les milieux locaux, favorisant les donations familiales.

Car cet essor du don de reliques s'explique par la densification des réseaux de patronage, ecclésiastiques et familiaux. De nombreuses opportunités permettent aux carmélites de multiplier et diversifier les réseaux d'approvisionnement en corps saints.

22. La famille de Longueville est très liée au Carmel depuis la fondation de l'ordre: Catherine d'Orléans a participé notamment à la fondation du carmel de Paris en 1603 (Morgain, 1995: 84).

23. Les Jésuites bénéficient depuis la découverte des catacombes d'un droit spécifique d'extraction. Ils développent une importante activité autour des reliques comme à Lille (Platelle, 1968 : 81-88). Luke Clossey a dressé un bilan de la politique des reliques de la Compagnie (Clossey, 2008: 220-230). 
Quelques grands donateurs donnent encore des reliques, comme la dominicaine romaine Maria Jozzi (Ticchi, 2016: 218) ou Louis Piffer d'Altisophen, dont la famille est très active parmi les gardes suisses pontificaux (Duhamelle, Baciocchi, 2016). Une élite urbaine, marchande et parlementaire, s'engage également dans la pratique du don, grâce notamment à un séjour à Rome ou à une proximité avec les milieux ecclésiastiques pontificaux. Par exemple, à Rouen, Philippe de Bojan de Castilly et Michel Certé, tous deux issus de familles de parlementaires normands, donnent respectivement sept et quatre corps saints romains obtenus lors d'un voyage à Rome au carmel rouennais, en 1671. Ils sont imités en 1687 par quatre autres familles de Normandie ${ }^{24}$. À Lyon, François Merle ${ }^{25}$ et Octave de la Bordésière, issus de familles consulaires, donnent six et sept reliques en 1685 (Grisard, 1887: 173 et 157), obtenues directement auprès du cardinal vicaire et de la sacristie pontificale. La famille de Rochebonne, cliente des Villeroy, donne en 1687 six reliques romaines (Gadille, 1983: 162).

C'est durant cette période que s'effectuent le plus grand nombre de dons familiaux. Les carmels étant bien ancrés dans la société urbaine, une deuxième génération de carmélites est à l'œuvre, souvent issue de la région. Charles Maignard ${ }^{26}$, Comte de Bernières, donne six reliques obtenues à Rome à deux de ses sœurs, carmélites à Rouen ${ }^{27}$. À Lyon, la pratique semble plus généralisée. La marquise de Pourcelet, Matthieu Pécoil, André Compain, Lucas Regnault de Bellescize ou encore Louis Carrel donnent durant cette période une relique au carmel qui accueille une fille ou une nièce. Tous ont séjourné à Rome ou ont occupé une fonction leur donnant accès, directement ou indirectement, aux reliques romaines ${ }^{28}$.

Les relations avec le milieu ecclésiastique sont également un facteur décisif dans les donations. Le réseau carme est encore une fois très actif. Le monastère de Lyon reçoit en 1680 des reliques en provenance du carmel d'Amiens en 1680 (Grisard, 1887: 154), puis du carmel de Pontoise en 1721, dans lequel Éléonore de Villeroy avait prononcé ses vœux (Grisard, 1887: 151). Le couvent rouennais reçoit lui des reliques de la part du carmel parisien du faubourg Saint-Jacques en 1680, mais aussi de la part de la branche masculine de l'ordre du Mont-Carmel. Philippe de Saint-Joseph, en 1672, achemine directement depuis Rome trois reliques. Victorin de Nymphe, vicaire provincial, en donne

24. A.D.S.M., 64H13, authentiques de reliques et validations épiscopales. Un tel investissement de la part des milieux parlementaires se retrouve à Bordeaux (Figeac et Le Mao, 2004) et à Grenoble (Coulomb, 2004).

25. Descendant d'un autre François Merle, prévôt des marchands de Lyon entre 1618 et 1621. 26. Ou Maignart.

27. A.D.S.M., 64H13, authentiques et vérification épiscopale. Barbe entre au carmel en 1643 sous le nom de Barbe de la Sainte-Trinité; Françoise prend l'habit en 1646 sous le nom de Françoise du Saint-Sacrement (Féron, 1930: 171)..

28. On retrouve dans cette liste plusieurs banquiers expéditionnaires à la Cour de Rome comme Pierre Compain ou Matthieu Pécoil. 
lui dix obtenues auprès du cardinal vicaire Carpegna à Rome ${ }^{29}$. D'autres ordres religieux interviennent également, tous issus de la réforme tridentine. Les carmélites nouent des contacts avec certains membres de ces ordres, qui interviennent comme prédicateur, supérieur ou encore directeur spirituel des carmélites. Si les feuillants continuent à donner des reliques ${ }^{30}$, les capucins sont également très actifs auprès du carmel lyonnais. Cette activité se concrétise par le don de deux lots de reliques des corps saints des catacombes en 1671 et 1692, grâce à l'entremise de prédicateurs prestigieux comme Alexandre de Lyon (Dompnier, 1990: 46), qui font le lien entre la maison romaine et Lyon. Même démarche à Rouen en 1700, où le frère capucin Hyacinthe de Paris donne deux reliques. Les Antonins, ordre plus ancien mais bien inséré dans les réseaux de distribution des corps saints, donnent trois lots de reliques au carmel de Lyon, en 1672, 1685 et 1693, motivés par des liens familiaux.

Et puis, après 1721, seuls deux dons pour le carmel de Lyon, dont celui effectué par François-Michel de Valadous en 1732, et un autre effectué par l'évêque d'Egée, suffragant du diocèse de Lyon, supérieur du couvent (Grisard, 1887: 173). La pratique du don de reliques disparait donc subitement. Comment l'expliquer?

\section{La société urbaine, les reliques, le don}

\section{Valeur des reliques, valeur du don}

Le don de reliques aux carmels de Lyon et Rouen recouvre, au XVII ${ }^{\mathrm{e}}$ siècle, des dynamiques autant sociales que spirituelles, qui expliquent en grande partie cette chronologie des arrivées. En tant qu'objets échangés, ou autrement dit, en tant que marchandises, les reliques peuvent être définies comme porteuses d'une valeur économique (Appadurai, 1986: 3). Cette valeur n'est pas interne à l'objet, mais un jugement produit à partir de lui par des sujets (Simmel, 1987). Cette valeur subjective est par définition fluctuante. En outre, les reliques ne sont pas un objet ordinaire. Relevant du domaine des choses sacrées, elles ne peuvent être vendues. Ce statut est défini dès le droit canon médiéval (Boiron, 2006 : 27). Cette inaliénabilité est confirmée par les décrets du concile de Trente (Alberigo, 1994: 1577). La valeur économique de la relique ne peut donc pas être, en droit, financière ${ }^{31}$. La valeur de la relique est liée à des considérations symboliques (ce que l'objet représente) et ontologiques (une réalité matérielle sacrée), qui en font un bien inestimable. Cette valeur repose ensuite sur la

29. A.D.S.M. $64 \mathrm{H} 13$, authentiques.

30. Le Provincial de la province Saint-Bernard, Nicolas de Saint-Augustin, visite régulièrement le carmel et donne deux reliques prélevées dans le trésor de l'abbaye Sainte-Marie de Pignerol, en 1669 (Grisard, 1887: 169-170).

31. Même si certains spécialistes de reliques trouvent, encore à l'époque moderne, le moyen de vendre leurs services Dans son article, Katie A. Harris montre qu'il existe une économie souterraine ( Underground Economy») des reliques des catacombes, avec de fréquents vols et ventes (Harris, 2014: 213). 
singularité de l'objet relique, insubstituable (Bartholeyns, 2012: 107), et sur son originalité, certifiée par les authentiques qui l'accompagnent. Si, durant la période médiévale, la valeur des reliques n'est quasiment pas remise en cause $^{32}$ par l'ensemble de la société, le $\mathrm{XVI}^{\mathrm{e}}$ siècle constitue une première période de crise. En effet, des théologiens comme Luther, Zwingli, Calvin, attaquent frontalement le culte des reliques, souvent sur le ton du sarcasme, pointant du doigt l'objet lui-même, émettant des doutes sur son authenticité ${ }^{33}$. Les protestants enlèvent toute valeur sacrale à la relique, n'y voyant qu'un reste humain (Harris, 2014: 221), et critiquent également la marchandisation des reliques ${ }^{34}$. Face à cette dévaluation symbolique et ontologique, à cette remise en cause des reliques et des dévotions qui leur sont associées, l'Église catholique, lors du concile de Trente, met en place des dispositifs visant à certifier l'authenticité des reliques, par la pratique systématique de l'authentique et du contrôle épiscopal. Ces mesures sont accompagnées par des écrits visant à justifier le culte des reliques par l'Écriture (Julia, 2009). Ainsi, dès le début $\mathrm{du} \mathrm{XVII}^{\mathrm{e}}$ siècle, les reliques renforcent leur position dans l'économie catholique du sacré, valorisées et régulées par les autorités ecclésiastiques. À ce même moment, les catacombes romaines commencent à livrer leur trésor, et les nouveaux ordres installés dans la catholicité désirent posséder des corps saints. C'est cette conjoncture qui explique en grande partie la valeur accordée au don de reliques, à cette période.

Il faut attendre le début du XVIII ${ }^{\mathrm{e}}$ siècle pour voir réapparaitre une nouvelle critique contre les reliques. Sous la plume des philosophes des Lumières, elles apparaissent alors comme l'exemple abouti de la superstition religieuse. Là se trouve peut-être une première explication à la disparition du don de reliques. Les milieux urbains, particulièrement sensibles aux courants de pensée des Lumières, délaissent cet objet de plus en plus controversé, trop ancré dans une matérialité jugée dangereuse, pour se consacrer à d'autres activités comme la charité, l'assistance et l'éducation (Gutton, 2004: 135).

À cette histoire intellectuelle de la valeur des reliques répond un versant matériel. La courbe des dons de reliques aux carmélites de Lyon et Rouen suit celle d'une diffusion plus générale à l'échelle de la catholicité. L'extraction et la mise en circulation de plus en plus massives de corps saints des catacombes sont allées bien au-delà de la demande, dévalorisant un objet qui, de fait, apparait progressivement comme de moins en moins extraordinaire. Au carmel de Rouen, ce sont vingt-trois dons de reliques qui sont reçus entre 1671 et 1700. Dans celui de Lyon, huit lots de corps saints arrivent entre 1683 et 1689. Dès lors, au tournant du XVIII ${ }^{\mathrm{e}}$ siècle, les carmels ont déjà constitué

32. Quelques théologiens émettent des critiques isolées comme Guibert de Nogent au XII ${ }^{\mathrm{e}}$ siècle (Hermann-Mascard, 1975: 107).

33. Un bilan de la critique protestante contre les reliques a été rédigé par Marie-Christine GomezPéraud (Gomez-Péraud, 1999).

34. Un polémiste réformé comme Antoine Marcourt associe notamment nombre de pratiques cultuelles catholiques aux échanges économiques de son époque (Marcourt, 1540). 
des trésors reliquaires dont il devient difficile de tenir l'inventaire exact. Le désir de reliques s'est sans doute affaibli à ce moment-là, alors que les reliques n'ont jamais été aussi accessibles.

Dans cette explication, si la valeur des reliques est fondamentale, la place $\mathrm{du}$ don et des acteurs qu'il met en jeu l'est également. Donner des reliques, au $\mathrm{XVII}^{\mathrm{e}}$ siècle, est un geste ambigu. En effet, le statut inaliénable des corps saints entraîne une nécessaire forme de don. Autre caractéristique, le don peut être partiel. La duchesse de Longueville donne, en 1659, un os de saint Hildevert, par fragmentation, le corps restant à Gournay. Enfin, les décrets tridentins insistent sur le monopole des ecclésiastiques sur les reliques. Au fil de la période, un laïc a de plus en plus de difficulté à en avoir la pleine propriété. Le 13 janvier 1672, le pape Clément X interdit aux personnes privées de posséder des reliques, qui doivent dès lors reposer dans un sanctuaire (Boiron, 2006: 109). Seuls les princes et prélats sont dorénavant autorisés à en garder pour un usage privé. Les laïcs, et ils sont de plus en plus nombreux pour la période étudiée, qui reçoivent un corps saint à Rome doivent donc, après 1672, le donner à une institution régulière ou séculière. Les carmélites provoquent parfois le don, comme dans le cas des reliques de saint Gérin, données par Anne de Souvré. En 1655, Marie de Brauvillier, abbesse de Montmartre, envoie des fragments d'os des martyrs de Montmartre "sur l'insistante prière que nous a faite la mère supérieure [du carmel de Rouen] » ${ }^{35}$. Mais pour la plupart des dons, le geste semble spontané et volontaire. Carlo Barberini, en 1656, envoie un os de saint Adrien, tiré des catacombes de Rome par Innocent X, au carmel de Lyon «de son bon gré » (Grisard, 1887: 167).

Dans l'économie du don, si la libéralité du donateur est donc visible dans les sources, elle se conjugue avec une forme de contre-don qu'est la prière des carmélites ${ }^{36}$. Donner une relique à un carmel ou en général à un établissement religieux, c'est s'assurer de prières en guise de remerciement. Ainsi le frère Poissuel, de l'ordre de Saint-Antoine, qui donne des reliques de sainte Dorothée et des saints Pie, Vital et Félicien à sa tante, carmélite à Lyon, lui demande dans sa lettre de "priez ces saincts pour moy, je vous en supplie » (Grisard, 1887: 157-158).

Si cette dimension spirituelle du don de reliques demeure présente tout au long de la période, la dimension sociale, elle, évolue. En effet, la multiplication des dons va de pair avec une évolution des profils des donateurs, surtout laïcs. Durant les deux premiers tiers du XVII ${ }^{\mathrm{e}}$ siècle, les dons sont le fait de personnalités importantes à l'échelle du royaume de France et de la catholicité, et l'exceptionnalité du geste répond à l'exceptionnalité du statut. Puis, à partir des années 1670, par une forme de mimétisme social, des milieux jugés de moindre qualité

35. A.D.S.M., 64H13, Reliques des martyrs de Montmartre, 1655.

36. Depuis l'ouvrage de Marcel Mauss (Mauss, 2007), le débat a été vif sur l'existence et la place du contre-don. On peut retenir avec Alain Testart (Testart, 2006), que le contre-don n'est pas exigible, mais dépend bien de la bonne volonté du receveur. Cependant, la contrepartie peut être l'objectif du donateur; ici la prière. 
commencent, eux-aussi, à donner des corps saints. Les élites urbaines lyonnaises et rouennaises s'approprient progressivement cette pratique du don ainsi que les réseaux d'accès aux reliques, jusque-là réservés à un cercle restreint, dans un processus d'aristocratisation par mimétisme de la noblesse (Mollat, 1979: 196). Cette appropriation est rendue possible par l'augmentation de la diffusion des reliques catacombaires. Et au fur et à mesure de sa généralisation, la valeur du don diminue, en même temps que la pratique se popularise.

Les deux conjonctures se rejoignent au début du XVII ${ }^{\mathrm{e}}$ siècle: la valeur de la relique diminue, le geste perd en honorabilité et en portée sociale. Pour les donateurs et pour les carmélites, la quête des corps saints perd en intérêt, et s'interrompt.

\section{Pratiques de distinctions}

Cette double évolution doit s'accompagner d'une contextualisation du don dans ces deux sociétés urbaines du Xvir ${ }^{\mathrm{e}}$ siècle. Donner des reliques aux carmélites peut, tout au long de cette période, revêtir une signification politique et sociale qui se superpose au spirituel. En effet, l'ordre carmélite est lié, dès son introduction en France, au milieu dévot (Meyer, 2001). Soutenir par sa libéralité un carmel, c'est affirmer son soutien et son appartenance à ce parti ${ }^{37}$. La pratique du don peut participer de la construction d'une sociabilité dévote, autour de dévotions et pratiques sociales communes, dans la lignée des fondateurs des carmels. À Rouen, le milieu dévot est assez actif, notamment dans le voisinage la compagnie du Saint-Sacrement. De nombreuses familles parlementaires y participent (Chaline, 1976: 129). Le don par Madame des Hameaux de sept reliques, dans la décennie 1630, s'inscrit dans cette dynamique. Son mari, Jacques Dyel de Miromesnil, sieur des Hameaux, conseiller au parlement de Normandie, est intendant du roi en 1636. Il pose, l'année précédente, la première pierre du couvent des carmes déchaux de Rouen. Un autre membre de cette Compagnie du Saint-Sacrement fait le lien avec le premier jansénisme normand. Charles Magnard de Bernières, fils d'un président du parlement, marié en 1638 avec Marie Amelot, fille d'un président de la cour des requêtes, développe des activités d'assistance, tout en léguant six corps saints des catacombes aux carmélites rouennaises après sa mort en 1662. Il joue un rôle important dans la conversion de la duchesse de Longueville au jansénisme. Il est également proche de la famille des ducs de Chaulnes, qui donne une relique au couvent lyonnais (Féron, 1930: 38 et 176). Le carmel de Lyon apparait lui comme particulièrement attaché à la famille des Neufville de Villeroy, proche du pouvoir royal, dont la plupart des membres ont leur sépulture dans la chapelle conventuelle. Dans une cité de Lyon en prise avec le pouvoir monarchique (Lignereux, 2004: 291-324), s'associer à une telle famille constitue bien un geste politique et religieux.

37. Sur le parti dévot, voir la synthèse de Jean-Pierre Gutton (Gutton, 2004). 
À l'échelle de la ville, le don de reliques permet également d'affirmer publiquement une réussite sociale. Le don de relique suppose des activités ou des relations qui permettent d'obtenir le précieux corps saints. Le cas des banquiers expéditionnaires lyonnais à la cour de Rome est assez représentatif. Le don dans le carmel de leur ville d'origine ressemble à un acte d'évergétisme, dans lequel la réussite personnelle d'un individu rejaillit sur l'ensemble de sa ville d'origine, qui gagne en sainteté.

À l'autre bout de la chaîne du don, l'accumulation du sacré participe aussi d'une stratégie de distinction pour les carmélites. À l'échelle de la ville, l'importance de leur trésor reliquaire distingue les carmels des autres couvents installés depuis peu. Ce trésor est souvent jugé exceptionnel par les contemporains. Ainsi, François Farin, prêtre rouennais, auteur d'une importante Histoire de Rouen publiée en 1668, mentionne cette spécificité:

Il y a peu de monastères à Rouen qui ayent le bonheur de posséder tant de saintes reliques que celui-ci (Farin, 1738: 114).

À Lyon, les différents Almanachs spirituels du XviII siècle signalent neuf reliques pour les carmélites ${ }^{38}$. Si le nombre parait peu élevé par rapport au total, le carmel lyonnais est l'un des grands centres reliquaires de la ville, avec la cathédrale Saint-Jean et l'église de l'Île-Barbe. Ces neuf reliques sont pour la plupart issues des dons effectués par les grands donateurs ${ }^{39}$. L'importance du trésor est un facteur important pour attirer des fidèles dans l'église conventuelle, ainsi que d'éventuels nouveaux donateurs. Les carmélites mettent ainsi en place des dispositifs à l'intérieur de leur sanctuaire pour rendre public et visible leur important trésor. À Lyon, les reliques sont réparties, d'après l'inventaire de 1782 (Grisard, 1887: 1445-151) dans quatorze reliquaires, placés dans le grand autel, la chapelle Saint-Nicolas et la sacristie. Les reliques sont ainsi visibles à la fois depuis le chœur des carmélites, mais aussi depuis la partie non cloitrée de l'église. Ce dispositif est renforcé par un calendrier de vingt-six fêtes autour des reliques, qui scandent l'année liturgique du couvent, avec à chaque fois des offices particuliers ${ }^{40}$. Ces deux carmels sont des centres spirituels dans leur ville respective, grâce aux reliques, malgré une situation périphérique dans l'espace urbain.

La constitution d'un trésor reliquaire permet donc aux carmels de Rouen et Lyon à la fois de pérenniser leur installation grâce au soutien des nombreux donateurs, tout en se distinguant dans le paysage religieux de la ville. Religieuses cloitrées, les carmélites sont pourtant très actives pour obtenir des

38. Nous sont parvenus les Almanachs spirituels des années 1698, 1705, 1729, 1733.

39. On retrouve le fragment de la croix donné par Philippe de l'Oratoire en 1642, le corps de saint Faustin obtenu du chapitre de Saint-Jean en 1628, un os de saint Adrien envoyé par Charles Barberini en 1656, un os de saint Victor donné par le carmel d'Amiens, un os de saint Érasme donné par la marquise de Pourcelet, le bras de sainte Clémence donné par Lucrèce Barberini, et enfin le corps de sainte Faustine envoyé par la dominicaine Maria Jozzi en 1682.

40. Semi-double, double et fête simple. 
reliques, multipliant les relations sociales : familiales, épistolaires, ecclésiastiques. Ainsi se créent les occasions de recevoir les corps saints, mobilisant tout un milieu urbain dévot, soucieux de montrer sa foi par des dévotions publiques (Chatellier, 1987: 163). La multiplication et la diversité des réseaux témoignent du besoin de posséder l'objet sacré chez les carmélites, pourtant souvent érigées dans l'historiographie comme l'exemple d'un renouveau spirituel fondé sur une intériorité dévotionnelle. Le désir des corps saints anime ainsi le cloître, suscitant d'importantes circulations. Les décrets tridentins, s'ils ont imposé un contrôle ecclésiastique sur l'objet relique, n'ont donc en rien limité son importance dans le catholicisme moderne.

\author{
Nicolas GUYARD \\ LabEx COMOD - LARHRA. \\ Université de Lyon - Université Lyon 2 \\ nxguyard@gmail.com
}

\title{
Bibliographie
}

Alberigo Giuseppe (éd.), 1994, Les conciles cecuméniques, Paris, Éditions du Cerf.

Appadurai Arjun, 1986, "Introduction: commodities and the politics of value”, Appadurai A. (ed.), The Social Life of Things: Commodities in Cultural Perspective, Cambridge, p. 3-63.

Baciocchi Stéphane, Boutry Philippe, Duhamelle Christophe, Fabre Pierre Antoine, Julia Dominique, 2010, «La distribution des corps saints des catacombes à l'époque moderne: de Rome aux nations ", Zuniga J.-P., Pratiques du transnational. Terrains, preuves, limites, Paris, La Bibliothèque du Centre de recherches historiques, p. 109-120.

Baciocchi Stéphane, Duhamelle Christophe, 2016, Reliques romaines. Invention et circulation des corps saints des catacombes à l'époque moderne, Rome, École Française de Rome.

Baciocchi Stéphane, Bonzon Anne, Julia Dominique, 2016, «De Rome au royaume de France: patronages, inscriptions spatiales et médiations sociales $\left(\mathrm{XVI}^{\mathrm{e}}-\mathrm{XVIII}^{\mathrm{e}} \mathrm{s}\right.$.). Introduction au dossier «France» », Baciocchi S., Duhamelle C., Reliques romaines. Invention et circulation des corps saints des catacombes à l'époque moderne, Rome, École Française de Rome, p. 413-458.

Bartholeyns Gil, 2012, «Les biens non substituables dans l'Occident médiéval », Techniques et cultures, 58, p. 104-121.

Baudiment Louis, 1934, François Pallu, principal fondateur de la Société des Missions étrangères, Paris, Gabriel Beauchesne.

Baudry Paul, 1878, Les Religieuses Carmélites à Rouen, Rouen, Imprimerie de H. Boissel. Nedict Philip, 1981, Rouen during the wars of religion, Cambridge, Cambridge University Press.

Boiron Stéphane, 2006, "Définition et statut juridique des reliques dans le droit canonique classique ", Deuffic J.-L., Reliques et sainteté dans l'espace médiéval, Saint-Denis, Pecia, p. 16-28. 
Boutry Philippe, 1979, "Les saints des Catacombes. Itinéraires français d'une piété ultramontaine (1800-1881) ", Mélanges de l'École française de Rome. Moyen Âge, Temps modernes, 91, p. 875-930.

Boutry Philippe, Fabre Pierre Antoine, Julia Dominique (éds.), 2009, Reliques modernes: cultes et usages chrétiens des corps saints des Réformes aux révolutions, Paris, Éditions de l'École des hautes études en sciences sociales.

Воzоку Edina, 2007, La politique des reliques de Constantin à Saint Louis. Protection collective et légitimation du pouvoir, Paris, Beauchesne.

Certeau de Michel, 1990, L'invention du quotidien. 1. Arts de faire, Paris, Gallimard.

Chaline Nadine-Josette, 1976, Histoire des diocèses de France. Rouen, Le Havre, Paris, Éditions Beauchesne.

Chatellier Louis, 1987, L’Europe des dévots, Paris, Flammarion.

Claverie Pierre-Vincent, 2009, "Les acteurs du commerce des reliques à la fin des croisades ", Le Moyen Âge, t. CXIV, no 3, p. 589-602.

Clossey Luke, 2008, Salvation and Globalization in the Early Jesuit Missions, Cambridge.

Condez Philippe, 2016, Trésors, mémoire, merveilles. Les objets des églises au Moyen Âge, Paris, Éditions de l'École des hautes études en sciences sociales.

Соulomв Clarisse, 2004, «Des parlementaires dévots. L'exemple de Grenoble au XVIII ${ }^{\mathrm{e}}$ siècle ", Chaline O., Sassier Y. (dirs.), Les Parlements et la vie de la cité (XVI ${ }^{e}$ XVIII ${ }^{e}$ siècle), Rouen, Publications de l'Université de Rouen, p. 301-321.

Davis Zemon Natalie, 2003, Essai sur le don dans la France du XVI siècle, Paris, Éditions du Seuil.

Diez Rodriguez José, 2005, Las Reliquias del Real Monasterio del Escorial, documentacion hagiografico, Madrid.

Dompnier Bernard, 1990, Enquête au Pays des Frères des Anges: Les Capucins de la Province de Lyon, aux XVII \& $\mathrm{XVIII}^{e}$ siècles, Clermont-Ferrand.

Duhamelle Christophe, 2016, "Candide, ou les voyages immobiles », Baciocchi S., Duhamelle C., Reliques romaines. Invention et circulation des corps saints des catacombes à l'époque moderne, Rome, École Française de Rome, p. 751-760.

Duhamelle Christophe, Baciocchi Stéphane, 2016, «Des gardes suisses à la frontière confessionnelle: apothéose et banalisation des corps saints des catacombes (Suisse, XVII-XVIII ${ }^{\mathrm{e}}$ siècles), Baciocchi S., Duhamelle C., Reliques romaines. Invention et circulation des corps saints des catacombes à l'époque moderne, Rome, École Française de Rome, p. 371-411.

Eriau Jean-Baptiste, 1929, L'ancien Carmel du faubourg Saint-Jacques (1604-1792), Paris, A. Picard éditeur.

FARIN François, 1738, Histoire de la ville de Rouen, Rouen, B. le Brun, in-12.

Féron Alexandre, 1930, La vie et les Euvres de Ch. Maignart de Bernières (1616-1662), Rouen, Lestringant.

Figeac Michel, Le Mao Caroline, 2004, «Le parlement de Bordeaux et la cité, de la Fronde à la veille de la Révolution ", Chaline O., Sassier Y. (dirs.), Les Parlements et la vie de la cité (XVI ${ }^{e}-\mathrm{XVIII}{ }^{e}$ siècle), Rouen, Publications de l'Université de Rouen, p. 249-276.

Gadille Jacques, 1983, Le diocèse de Lyon, Paris, Beauchesne. 
Gana Maddalena, 2005, "Reliquie e nobildonne nella Roma Barocca », Sanctorum, 2, p. 111-120.

Geary Patrick J., 1986, “Sacred Commodities: The Circulation of Medieval Relics”, Appadurai A. (ed.), The Social Life of Things: Commodities in Cultural Perspective, Cambridge, p. 169-191.

Geary Patrick J., 1990, Furta Sacra: Thefts of Relics in the Central Middle Ages, Princeton. Ghilardi Massimiliano, 2003, «Le catacombe di Roma nel Medioevo. Riflessioni recenti e prospettivi di ricerca ", Subterranea civitas. Quattro studi sulle catacombe romane dal medioevo all'età moderna, Rome, p. 17-41.

GiORDANi Roberto, 2007, «La scoperta della catacomba sotta la vigna Sanchez e la nascita degli studi d'antichità christiane ", Rivista di archaologia cristiana, 83, p. 277-315.

Gomez-Géraud Marie-Christine, 1999, Le crépuscule du grand voyage: les récits de pèlerins à Jérusalem (1458-1612), Paris, Honoré Champion.

GRISARD Jacques-Jules, 1887, Documents pour servir à l'histoire du couvent des carmélites de Notre-Dame de la Compassion de Lyon, Lyon.

GutTon Jean-Pierre, 2004, Dévots et société au XVII siècle. Construire le ciel sur la Terre, Paris, Belin.

Harris A. Katie, 2014, "Gift, Sale, and Theft: Juan de Ribera and the Sacred Economy of Relics in the Early Modern Mediterranean”, Journal of Early Modern History, 18, p. 193-226.

HermanN-Mascard Nicole, 1975, Les reliques des saints. Formation coutumière d'un droit, Paris, Klincksieck.

Hours Bernard, 1987, Madame Louise, princesse an Carmel:1737-1787, Paris, Éditions du Cerf.

Hours Bernard (dir.), 2001, Carmes et carmélites en France du XVII siècle à nos jours, actes du colloque de Lyon des 25 au 26 septembre 1997, Paris, Éditions du Cerf.

Julia Dominique, 2009, "L'Église post-tridentine et les reliques. Tradition, controverse et critique (XVI ${ }^{\mathrm{e}}-\mathrm{XVII}^{\mathrm{e}}$ siècle) », Boutry P., Fabre P. A., Julia D. (éds.), Reliques modernes: cultes et usages chrétiens des corps saints des Réformes aux révolutions, Paris, Éditions de l'École des hautes études en sciences sociales, p. 69-120.

Lazure Guy, 2009, «Posséder le sacré. Monarchie et identité dans la collection de reliques de Philippe II à l'Escorial », Boutry P., Fabre P. A., Julia D. (éds.), Reliques modernes: cultes et usages chrétiens des corps saints des Réformes aux révolutions, Paris, Éditions de l'École des hautes études en sciences sociales, p. 371-404.

Legner Anton, 2003, Kölner Heilige und Heiligtümer: ein Jahrtausend europäischer Reliquienkultur, Köln, Greven.

Le Hénand Françoise, 2009, "Les translations de reliques en France au XVII siècle ", Boutry P., Fabre P. A., Julia D. (éds.), Reliques modernes: cultes et usages chrétiens des corps saints des Réformes aux révolutions, Paris, Éditions de l'École des hautes études en sciences sociales, p. 313-370.

Lignereux Yann, 2003, Lyon et le roi: de la "bonne ville" à l'absolutisme municipal, Seyssel, Champ Vallon.

Marcourt Antoine, 1540, Le Livre des Marchands.

Martin Jean-Baptiste, 1909, Histoire des églises et chapelles de Lyon, Lyon. 
Mauss Marcel, 2007, Essai sur le don, Forme et raison de l'échange dans les sociétés archaïques, Paris, Presses universitaires de France.

Mellot Jean-Dominique, 1994, Histoire du Carmel de Pontoise, Paris, Desclée de Brouwer.

Meyer Frédéric, 2001, "Carmélites et laïques dévots: l'exemple des fondations comtadines ", Hours B. (dir.), Carmes et carmélites en France du XVII siècle à nos jours, Paris, p. 7-18.

Mollat Michel, 1979, Histoire de Rouen, Toulouse, Privat.

Morgain Stéphane-Marie, 1995, Pierre de Bérulle et les Carmélites de France, Paris, Éditions du Cerf.

MOUlinier Laurence, 1993, «Élisabeth, Ursule et les Onze mille vierges: un cas d'invention de reliques à Cologne au XII ${ }^{\mathrm{e}}$ siècle ", Médiévales, 22-23, p. 173-186.

Osborne John, 1999, "Politics, Diplomacy and the Cult of Relics in Venice and Northern Adriatic in the First Half of the Nineth Century", Early Medieval Europe, 5, $\mathrm{n}^{\circ} 3$, p. 369-386.

Pierre Benoist, 2006, La bure et le sceptre: la congrégation des Feuillants dans l'affirmation des États et des pouvoirs princiers, vers 1560-vers 1660, Paris, Publications de la Sorbonne.

Platelle Henri, 1968, Les Chrétiens face au miracle. Lille au XVII e siècle, Paris, Éditions du Cerf.

Roullet Antoine, 2015, Corps et pénitence: les carmélites déchaussées espagnoles (ca 1560-ca 1640), Madrid, Casa de Velazquez.

Serouet Pierre, 1971, Quintanaduenas. Lettres de Jean de Brétigny, 1556-1634, Louvain, Bibliothèque de la Revue d'histoire ecclésiastique.

Simmel Georg, 1987, Philosophie de l'argent, Paris, Presses universitaires de France.

Testart Alain, 2006, Des dons et des dieux: anthropologie religieuse et sociologie comparative, Paris, Éditions Errance.

Ticchi Jean-Marc, 2016, «Mgr sacriste et la distribution des reliques des catacombes dans l'espace italien ", Baciocchi S., Duhamelle C., Reliques romaines. Invention et circulation des corps saints des catacombes à l'époque moderne, Rome, École Française de Rome, p. 175-223.

VACHET Adolphe, 1895, Les anciens couvents de Lyon, Lyon, E. Vitte.

\section{Sanctifier le cloître. Les dons de reliques aux carmélites de Lyon et Rouen à l'époque moderne}

La fondation des carmels dans les villes de Lyon et de Rouen au début du XVII siècle entraîne un besoin de sacralité. Les nouveaux monastères doivent être dotés de reliques, pour sanctifier le sanctuaire, mais aussi attirer des fidèles, favoriser les donations en tout genre. Pour cela, les carmélites mobilisent de multiples réseaux, suivant des logiques spatiales et sociales qui s'amplifient jusqu'à la fin du siècle. Bien que cantonnées dans leur cloître, elles obtiennent des reliques variées, tirées des différents centres de distribution, grâce aux relais efficaces de donateurs, laïcs et ecclésiastiques. Les carmélites amassent ainsi des trésors considérables, au centre de la société urbaine de leur époque.

Mots clés: reliques, carmélites, don, circulation, catholicisme. 


\section{Sanctification of the cloister. Gifts of relics to the Carmelites of Lyon and Rouen in the modern period}

The foundation of carmels in the cities of Lyon and Rouen in the beginning of the XVII ${ }^{\text {th }}$ century brings a need for sacred. The new monasteries must possess relics in order to sanctify the sanctuary, but also attract the believers and encourage all kinds of donations. With this aim in mind, the Carmelites muster various networks, following spatial and social logics which grow stronger until the end of the century. Though they are confined in their cloister, they manage to obtain various relics, through the different centres of distribution and thanks to the efficient mediation of donators, lays and ecclesiastics. The Carmelites thus accumulate substantial treasures, acting as true reliquaries at the heart of the urban society of their time.

Key words: relics, Carmelites, gift, circulation, catholicism.

\section{Santificar el claustro. reliquias de donaciones a Carmelite Lyon y Rouen en la era moderna}

La fundación de los carmeles en las ciudades de Lyon y Ruan en el principio del siglo XVII entrena una necesidad de sagrado. Los nuevos monasterios deben dotarse con reliquias para santificar el santuario, pero también para atraer a los fieles y favorecer las donaciones de todos tipos. Para eso, las Carmelitas recurren a varias redes, según lógicas espaciales y sociales que se amplifican hasta el final del siglo. Aunque están encerradas en su claustro, consiguen varias reliquias obtenidas en los varios centros de distribución, a través del intermediario efectivo de donadores, laicos y eclesiásticos. De esta forma, las Carmelitas acumulan tesoros sustanciales, actuando como verdaderas relicarias al centro de la sociedad urbana de su época.

Palabras claves: reliquias, Carmelitas, donación, circulación, catolicismo. 